Chapter 32.

\title{
Onion, Leek, and Chive Production in Florida
}

\section{S.M. Olson, W.M. Stall, K.L. Pernezny, S.E. Webb}

\section{BOTANY}

\section{Nomenclature}

Family - Alliaceae (Amaryillidaceae)

Onion - Allium cepa

Leek - Allium ampeloprasum

Chive - Allium schoenoprasum

\section{Origin}

It is believed that all of the edible onions and their relatives are native to Asia.

\section{Related Species}

Garlic, shallot, and various exotic onions also are members of the Alliaceae family. Many important ornamentals also are included in this family.

\section{VARIETIES}

Onion and leek varieties grown in Florida are listed below $(\mathrm{H}=$ hybrid):

\author{
Onion \\ Caramelo $(\mathrm{H})$ \\ Dessex $(\mathrm{H})$ \\ Georgia $(\mathrm{H})$ \\ Granex $33(\mathrm{H})$ \\ Linda Vista $(\mathrm{H})$ \\ Savannah Sweet $(\mathrm{H})$ \\ Sugar Belle $(\mathrm{H})$ \\ Sweet Success $(\mathrm{H})$ \\ Bunching Onion \\ Perfecto Blanco \\ Tokyo Long White \\ White Portugal \\ Leeks (Fig. 32-1) \\ King Richard \\ Tivi \\ Verina
}

\section{SEEDING AND PLANTING}

Planting dates and seeding information are given in Table 1.

Table 1. Seeding and planting information for onion and allies.

\begin{tabular}{|lll||}
\hline Planting dates & Seeded & Transplanted \\
\hline \hline North Florida & Mid Sept - mid Nov & Nov - Jan \\
Central Florida & Oct & Dec - Jan \\
South Florida & Oct & Dec - Jan \\
Planting information & & \\
Distance between rows (in) ${ }^{1}$ & $14-18$ & $14-18$ \\
Distance between plants (in) & $3-4$ & $4-6$ \\
Seeding depth (in) & $0.25-0.5$ & - \\
Seed per acre (lb) & $3-4$ & 1.0 \\
Days to maturity & $100-130$ & $100-130^{2}$ \\
Plant populations (acre) & 149,343 & 112,123 \\
\hline \hline${ }^{2}$ Traditionally 14 inches between rows, 4 inches between plants. \\
${ }^{2}$ From time of field setting. & \\
\hline
\end{tabular}




\section{FERTILIZER AND LIME}

For unmulched onions with subsurface or sprinkler irrigation, broadcast all $\mathrm{P}_{2} \mathrm{O}_{5}$ micronutrients, and 20 to $25 \%$ of $\mathrm{N}$ and $\mathrm{K}_{2} \mathrm{O}$ before planting (Fig. 32-2). Apply remaining $\mathrm{N}$ and $\mathrm{K}_{2} \mathrm{O}$ in split applications through the season up to bulb initiation. Overfertilization with $\mathrm{N}$ during bulbing can lead to double bulbs and split bulbs.

For mulched onions, incorporate all fertilizer in bed prior to mulching (Fig. 32-3). For mulched onions with subsurface irrigation, incorporate only 20 to $25 \%$ of the $\mathrm{N}$ and $\mathrm{K}_{2} \mathrm{O}$. Place remaining $\mathrm{N}$ and $\mathrm{K}_{2} \mathrm{O}$ in bands in grooves 2 to 3 inches deep between rows on bed (see Table 2).

\section{IRRIGATION}

Irrigation requirements of onions can be estimated from crop coefficients given in Tables 4 to 6 in Chapter 8, Principles and Practices of Irrigation Management for Vegetables. Irrigation requirements of leek and chives are expected to be similar to those shown for onions. Peak water use during rapid growth and development stages will be about $95 \%$ of ETo. Water use will continue at this rate for plants that are harvested green, while water use will drop to about $75 \%$ of ETo for onions that are allowed to dry before harvest. See Table 3 in Chapter 8, Principles and Practices of Irrigation Management for Vegetables for estimates of ETo in inches per day and gallons per acre per day for Florida climate conditions.

For optimum production, irrigations should be scheduled to maintain adequate soil moisture throughout the growing season for plants that are harvested green. For onions that are to be harvested dry, irrigation can be discontinued after full growth has been obtained and the plants begin to dry. For drip and sprinkler irrigation, small, frequent applications should be scheduled to avoid water and nutrient losses below the plant root zone. For seepage irrigation, the field water table should be maintained sufficiently high that soil moisture is adequate but not excessively wet in the plant root zone throughout the growing season or until the plants are allowed to begin to dry for harvest.

\section{PLANT TISSUE ANALYSIS}

Plant tissue analysis information for onion and allies is given in Table 3 . The analysis was done prior to bulbing, using the most recently matured leaf.

\section{WEED MANAGEMENT}

Herbicides labeled for weed control in onions and allies are listed in Table 4.

\section{DISEASE MANAGEMENT}

The chemicals approved for disease management in onion and leek are listed in Table 5.

\section{INSECT MANAGEMENT}

Table 6 outlines the insecticides approved for use on insects attacking onion and allies.

Table 2. Soil test and fertilizer recommendations for mineral soils for onion and allies. ${ }^{1}$

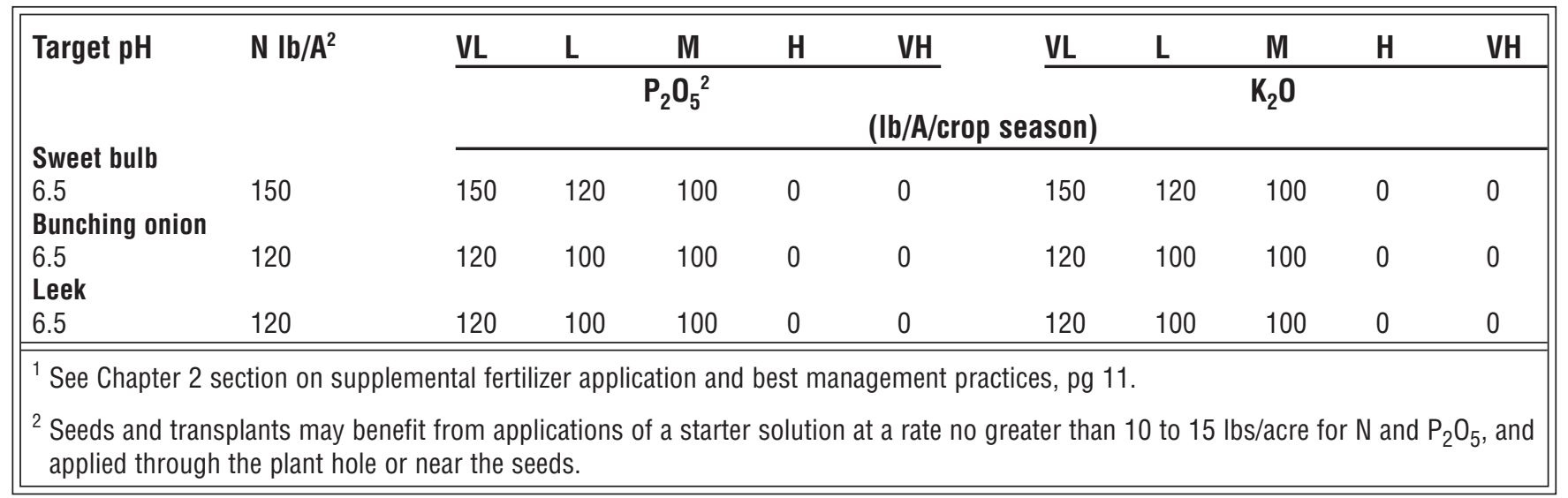

Table 3. Plant tissue analysis just prior to bulbing for onion and allies. Dry wt. basis.

\begin{tabular}{|c|c|c|c|c|c|c|c|c|c|c|c|}
\hline \multirow[b]{2}{*}{ Status } & $\mathbf{N}$ & $\mathbf{P}$ & K & $\mathrm{Ca}$ & $\mathrm{Mg}$ & $S$ & $\mathrm{Fe}$ & Mn & $\mathrm{Zn}$ & B & $\mathrm{Cu}$ \\
\hline & \multicolumn{6}{|c|}{ Percent } & \multicolumn{5}{|c|}{ Parts per million } \\
\hline Deficient & $<2.0$ & 0.2 & 1.5 & 0.6 & 0.15 & 0.2 & 50 & 10 & 15 & 10 & 5 \\
\hline Adequate range & $2.0-3.0$ & $0.2-0.5$ & $1.5-3.0$ & $0.6-0.8$ & $0.15-0.30$ & $0.2-0.6$ & $50-100$ & $10-20$ & $15-20$ & $10-25$ & $5-10$ \\
\hline High & $>3.0$ & 0.5 & 3.0 & 0.8 & 0.30 & 0.6 & 100 & 20 & 20 & 25 & 10 \\
\hline Toxic (>) & & & & & & & & & & 100 & \\
\hline
\end{tabular}


Table 4. Chemical weed controls: onions, leek, garlic \& shallot.

\begin{tabular}{|c|c|c|c|c|}
\hline \multirow[b]{2}{*}{ Herbicide } & \multirow[b]{2}{*}{ Labeled crops } & \multirow{2}{*}{$\begin{array}{l}\text { Time of } \\
\text { application to crop }\end{array}$} & \multicolumn{2}{|c|}{ Rate (Ibs. Al./Acre } \\
\hline & & & Mineral & Muck \\
\hline $\begin{array}{l}\text { Bensulide } \\
\text { (Prefar 4E) }\end{array}$ & onions (dry bulb), garlic, shallots & $\begin{array}{l}\text { Preplant } \\
\text { Preemergence }\end{array}$ & $5-6$ & -- \\
\hline
\end{tabular}

Remarks: Preplant incorporate to depth of 1-2 inches in well worked soil. Apply preemergence and irrigate to incorporate. With overhead irrigation, wet soil at least 2-4 inches deep. For furrow irrigation thoroughly wet the entire bed top. Controls many grass species.

\begin{tabular}{llll}
\hline \hline $\begin{array}{l}\text { Bromoxynil } \\
\text { (Buctril) }\end{array}$ & Onions & Preemergence & $0.25-0.375$ \\
Garlic & Postemergence & --
\end{tabular}

Remarks: Apply to onions before planting until just prior to crop emergence (onions only). Postemergence apply to onions which have 2 to 5 true leaves or to garlic after emergence but before 12 inches in height. Controls several broadleaf weeds such as lambsquarter, smartweed, morningglory, spiny pigweed, ragweed, etc. (see label). When they are small, do not exceed 4 leaf stage, or less than 2 inches in height. Use at least 50 to 70 gallons of water per acre for application. Soil and onion foliage must be dry at time of applications. Waxy coating on onion leaves reduces chances for injury. Onion varieties vary in sensitivity. Use on a trial basis.

$\begin{array}{llll}\text { Carfentrazone } & & \text { Preplant } & 0.031 \\ (\text { Aim) } & \text { (All) } & \text { Directed-hooded } & 0.031\end{array}$

Row-middles

Remarks: Aim may be applied as a preplant burndown treatment and/or as a post-directed hooded application to row middles for the burndown of emerged broadleaf weeds. May be tank mixed with other registered herbicides. May be applied at up to $2 \mathrm{oz}(0.031 \mathrm{lb}$ ai). Use a quality spray adjuvant such as crop oil concentrate (coc) or non-ionic surfactant at recommended rates.

\begin{tabular}{llll}
\hline \hline Clethodim & Onions (dry bulb) & Postemergence & $0.94-.125$ \\
(Select) & Garlic & & $.094-.128$ \\
& Shallots (dry bulbs) & &
\end{tabular}

Remarks: Material is a selective postemergence herbicide for control of annual and perennial grasses. Always use a crop oil concentrate at $1 \% \mathrm{v} / \mathrm{v}$ in the finished spray volume should range from 5 to 40 gallons per acre. Rates range from 6 to 8 oz. Product per acre for annual grasses up to $16 \mathrm{oz}$. Product for perennial grasses. Do not apply within 45 days of harvest.

\begin{tabular}{lll}
\hline DCPA (Dacthal W-75) & Onions, Garlic & Preemergence \\
& & Posttransplanting or At layby
\end{tabular}

Remarks: Controls germinating annuals. Incorporate 0.5 to 1.0 inch with overhead irrigation or shallow cultivation. Apply layby treatment to weed-free field up to 14 weeks after planting at rates not exceeding $10.5 \mathrm{lbs}$. ai./acre per season. In Hastings area, onions may be injured with single applications exceeding $6.0 \mathrm{lbs}$. ai./acre. Note precautions of planting non-registered crops within 8 months.

\begin{tabular}{lllll}
\hline \hline Fluazifop-p (Fusilade) & Dry bulb onions, garlic & Postemergence & 0.188 & 0.188
\end{tabular}

Remarks: Controls actively growing grass weeds. A total of $48 \mathrm{oz}$. product may be applied to the crop per season. Rates for the control of actively growing grass species at specific growth stages are specified on the label. Depending on the species, the growth stage for best control ranges from the 3- to 8-leaf stage. Use oil concentrates or non-ionic surfactants in the spray mixture. A pre-harvest interval of 45 days must be maintained.

\begin{tabular}{|lll||}
\hline \hline Glyphosate & Chemical fallow & $0.3-1.0$ \\
(Roundup, Durango & Preplant, pre emergence, & \\
Touchdown, Glyphomax) & Pre transplant & \\
\hline
\end{tabular}

Remarks: Roundup, Glyphomax and Touchdown have several formulations. Check the label of each for specific labeling directions.

\begin{tabular}{llll}
\hline \hline Oxyfluorfen (Goal 2XL) & Dry bulb onions & Early postemergence & 0.12 \\
(Goaltender) & Posttransplant & 0.5 & ---
\end{tabular}

Remarks: Spray when seeded onions have 2 fully developed true leaves. On transplanted onions spray as soon after transplanting as practical. Necrotic lesions, twisting or stunting of onion plants can occur if applications are made during cool, wet weather or prior to the full development of the true leaves on the onion plant. Multiple applications may be made. Do not apply within 60 days of harvest.

\begin{tabular}{|lllc|}
\hline \hline $\begin{array}{l}\text { Paraquat } \\
\text { (Gramoxone Intron) }\end{array}$ & Seeded onions: Green or & Preplant & $0.63-0.94$ \\
(Firestorm) & dry bulb \& Garlic & Preemergence & $0.63-0.94$ \\
\hline
\end{tabular}

\section{(Firestorm)}

Remarks: Apply as a broadcast treatment prior to, during or after seeding, but before emergence of the crop for control of emerged weeds. Weeds and grasses emerging after treatment will not be controlled. Crop plants emerged at the time of application will be damaged. Use a non-ionic surfactant with application. Do not apply within 60 days of harvest.

\begin{tabular}{|c|c|c|c|}
\hline $\begin{array}{l}\text { Pendimethalin } \\
\text { (Prowl 3.3 EC) }\end{array}$ & Dry Bulb Onions & $\begin{array}{l}\text { Preemergence (muck only) } \\
\text { Postemergence or post-transplant }\end{array}$ & $0.5-0.75$ \\
\hline
\end{tabular}

Remarks: In mineral soils, apply as broadcast treatment when onions have 2 to 9 true leaves at a rate of 1.2 to 1.8 pts. In muck soils may be applied sequentially as follows: Preemergence through loop stage (2.4 to $4.8 \mathrm{pts} / \mathrm{A}$ ); Early postemergence (2 to 6 true leaf stage) 3.6 to $4.8 \mathrm{pts} / \mathrm{A}$; Late postemergence (6 to 9 true leaf stage) 3.6 to $4.8 \mathrm{pts} / \mathrm{A}$. Do not apply more than $14.4 \mathrm{pts} / \mathrm{A}$ per growing season on muck soils. Do not apply preemergence through loop stage if heavy rains are expected or severe crop injury may result. If irrigating after application, do not irrigate in excess of 0.5 inch. Do not apply within 45 days of harvest.

\begin{tabular}{|llll|}
\hline \hline Sethoxydim (Poast) & $\begin{array}{l}\text { Bulb vegetables: all onions, } \\
\text { dry bulb and bunching, garlic, leeks }\end{array}$ & Postemergence & .187 \\
\hline
\end{tabular}

Remarks: For control of actively growing grass weeds. Always add a crop oil concentrate at a rate of 2 pts./acre. Do not apply within 30 days of harvest. A general use rate of 1 pt. material may be used. Do not apply more than 4.5 pts. per acre in one season. 
Table 5. Disease management for onion.

\begin{tabular}{|c|c|c|c|c|c|c|}
\hline Chemical (a.i.) & $\begin{array}{l}\text { FRAC } \\
\text { Group1 }\end{array}$ & $\frac{\text { Maximum }}{\text { Application }}$ & $\frac{\text { Rate/Acre/ }}{\text { Season }}$ & $\begin{array}{l}\text { Min. Days } \\
\text { to Harvest }\end{array}$ & $\begin{array}{l}\text { Pertinent Diseases } \\
\text { or Pathogens }\end{array}$ & Remarks $^{2}$ \\
\hline $\begin{array}{l}\text { Ridomil Gold } 4 \text { EC } \\
\text { (mefenoxam) }\end{array}$ & 4 & $\begin{array}{l}1 \text { pt./trted } \\
\text { acre }\end{array}$ & & & Pythium seedling blight & $\begin{array}{l}\text { Apply at seeding in a } 7-12 \text { " band } \\
\text { on soil over seed furrow }\end{array}$ \\
\hline Manex $4 \mathrm{~F}$ (maneb) & M3 & 2.4 qts & 24 qts $^{1}$ & 7 & $\begin{array}{l}\text { Botrytis leaf blight } \\
\text { Purple blotch }\end{array}$ & Do not apply to exposed bulbs \\
\hline $\begin{array}{l}\text { Maneb } 80 \text { WP } \\
\text { (maneb) }\end{array}$ & M3 & $3 \mathrm{lbs}$ & $30 \mathrm{lbs}^{2}$ & 7 & $\begin{array}{l}\text { Botrytis leaf blight } \\
\text { Purple blotch }\end{array}$ & Do not apply to exposed bulbs \\
\hline $\begin{array}{l}\text { Botran } 75 \mathrm{~W} \\
\text { (dichloran) }\end{array}$ & 14 & $51 / 3 \mathrm{lbs}$ & $51 / 3 \mathrm{lbs}$ & & Botrytis & Limit is 1 appl./crop \\
\hline $\begin{array}{l}\text { Botran } 5 \mathrm{~F} \\
\text { (dichloran) }\end{array}$ & 14 & $13 / 5$ qts & 2 qts & 14 & Botrytis & $\begin{array}{l}\text { Do not plant spinach as a follow } \\
\text { up crop }\end{array}$ \\
\hline $\begin{array}{l}\text { Penncozeb, Dithane } \\
\text { M45, or Manzate } 80 \\
\text { WPs (Dry bulb only) } \\
\text { (mancozeb) }\end{array}$ & M3 & $3 \mathrm{lbs}$ & $30 \mathrm{lbs}$ & 7 & $\begin{array}{l}\text { Botrytis leaf blight } \\
\text { Purple blotch }\end{array}$ & Do not apply to exposed bulbs \\
\hline $\begin{array}{l}\text { Dithane F-45 or } \\
\text { Manex II } 4 \text { FLs } \\
\text { (Dry bulb only) } \\
\text { (mancozeb) }\end{array}$ & M3 & 2.4 qts & 24 qts & 7 & $\begin{array}{l}\text { Botrytis leaf blight } \\
\text { Purple blotch }\end{array}$ & Do not apply to exposed bulbs \\
\hline $\begin{array}{l}\text { Rovral } 75 \text { WG } \\
\text { (Dry bulb only) (ipro- } \\
\text { dione) }\end{array}$ & 2 & $1 \mathrm{lb}$ & $5 \mathrm{lbs}$ & 7 & $\begin{array}{l}\text { Botrytis leaf blight } \\
\text { Purple blotch }\end{array}$ & Limit 5 appl./season \\
\hline $\begin{array}{l}\text { Maneb } 75 \text { DF } \\
\text { (maneb) }\end{array}$ & M3 & $3 \mathrm{lbs}$ & $32 \mathrm{lbs}^{3}$ & 7 & $\begin{array}{l}\text { Botrytis leaf blight } \\
\text { Purple blotch }\end{array}$ & Do not apply to exposed bulbs \\
\hline $\begin{array}{l}\text { Equus } 720 \text { or Echo } \\
7206 \text { FLs (chloro- } \\
\text { thalonil) }\end{array}$ & M5 & $2 \mathrm{pts}^{4}$ & $20 \mathrm{pts}$ & 75 & $\begin{array}{l}\text { Botrytis leaf blight } \\
\text { Purple blotch }\end{array}$ & $\begin{array}{l}\text { Limit is } 3 \text { appl./crop for green } \\
\text { bunching onions, leeks or shal- } \\
\text { lots }\end{array}$ \\
\hline $\begin{array}{l}\text { Bravo Ultrex or Echo } \\
\text { Ultimate } 82.5 \text { WDGs } \\
\text { (chlorothalonil) }\end{array}$ & M5 & $1.8 \mathrm{lbs}^{6}$ & $\begin{array}{l}18.2 \mathrm{lbs} \\
(8.2 \mathrm{lbs} \\
\text { for green } \\
\text { onions, } \\
\text { shallots \& } \\
\text { leeks) }\end{array}$ & 7 & $\begin{array}{l}\text { Botrytis leaf blight } \\
\text { Purple blotch }\end{array}$ & $\begin{array}{l}\text { Limit is } 3 \text { appl./crop for green } \\
\text { bunching onions, leeks or shal- } \\
\text { lots }\end{array}$ \\
\hline $\begin{array}{l}\text { Penncozeb, Dithane, } \\
\text { or Manzate } 75 \text { DFs } \\
\text { (mancozeb) }\end{array}$ & M3 & $3 \mathrm{lbs}$ & $32 \mathrm{lbs}$ & 7 & $\begin{array}{l}\text { Botrytis leaf blight } \\
\text { Purple blotch }\end{array}$ & Do not apply to exposed bulbs \\
\hline $\begin{array}{l}\text { Bravo Weather Stik } 6 \\
\text { F (chlorothalonil) }\end{array}$ & M5 & $2 \mathrm{pts}^{4}$ & $9 \mathrm{pts}$ & 7 & $\begin{array}{l}\text { Botrytis leaf blight } \\
\text { Purple blotch }\end{array}$ & $\begin{array}{l}\text { Limit is } 3 \text { appl./crop for green } \\
\text { bunching onions, leeks or shal- } \\
\text { lots }\end{array}$ \\
\hline $\begin{array}{l}\text { Pristine } 38 \text { WG } \\
\text { (pyraclostrobin/ } \\
\text { boscalid) }\end{array}$ & $11+7$ & $18.5 \mathrm{ozs}$ & 111 ozs & 7 & $\begin{array}{l}\text { Botrytis leaf blight } \\
\text { Purple blotch } \\
\text { Stemphyllium leaf blight }\end{array}$ & $\begin{array}{l}\text { Limit is } 6 \text { appl./crop \& alternate } \\
\text { chemistry }\end{array}$ \\
\hline $\begin{array}{l}\text { Quadris Opti } 0.5 \& 5 \\
\text { FL (azoxystrobin) }\end{array}$ & 11 & $3.2 \mathrm{pts}$ & $11.1 \mathrm{pts}$ & 14 & $\begin{array}{l}\text { Purple blotch } \\
\text { Botrytis }\end{array}$ & $\begin{array}{l}\text { Limit is } 3 \text { appl./crop \& alternate } \\
\text { chemistry. For green bunching, } \\
\text { leeks \& shallots }\end{array}$ \\
\hline $\begin{array}{l}\text { Endura } 70 \text { WP } \\
\text { (boscalid) }\end{array}$ & 7 & $6.8 \mathrm{ozs}$ & $41 \mathrm{ozs}$ & 7 & $\begin{array}{l}\text { Purple blotch } \\
\text { Botrytis }\end{array}$ & $\begin{array}{l}\text { Limit is } 6 \text { appl/crop \& alternate } \\
\text { chemistry }\end{array}$ \\
\hline $\begin{array}{l}\text { Acrobat 50WP } \\
\text { (dimethomorph) }\end{array}$ & 40 & $6.40 z$ & $320 z$ & 0 & Downy mildew & $\begin{array}{l}\text { Must be applied in a tank mix with } \\
\text { another fungicide active against } \\
\text { downy mildew. Do not make more } \\
\text { than } 2 \text { sequential appl. }\end{array}$ \\
\hline $\begin{array}{l}\text { Phostrol } 54 \% \text { Salt } \\
\text { (salts of phosphorus } \\
\text { acid) }\end{array}$ & 33 & $3.75 \mathrm{pt}$ & $26.25 \mathrm{pt}$ & 0 & Powdery mildew & \\
\hline $\begin{array}{l}\text { Aliette WDG (fos- } \\
\text { etyl-Al) }\end{array}$ & 33 & $3.0 \mathrm{lb}$ & $21 \mathrm{lb}$ & 7 & $\begin{array}{l}\text { Downy mildew } \\
\text { Purple blotch }\end{array}$ & \\
\hline
\end{tabular}


Table 5. Continued.

\begin{tabular}{|c|c|c|c|c|c|c|}
\hline Chemical (a.i.) & $\begin{array}{l}\text { FRAC } \\
\text { Group } 1\end{array}$ & $\frac{\text { Maximum }}{\text { Application }}$ & $\frac{\text { Rate/Acre/ }}{\text { Season }}$ & $\begin{array}{l}\text { Min. Days } \\
\text { to Harvest }\end{array}$ & $\begin{array}{l}\text { Pertinent Diseases } \\
\text { or Pathogens }\end{array}$ & Remarks $^{2}$ \\
\hline $\begin{array}{l}\text { Helena Prophyt } \\
54.5 \% \text { Salt (potas- } \\
\text { sium phosphite) }\end{array}$ & 33 & 2 qt & & 0 & Powdery mildew & \\
\hline $\begin{array}{l}\text { Various copper } \\
\text { compounds (see ind. } \\
\text { Labels), including } \\
\text { Basic Copper 53, } \\
\text { Champ, COC, Copper } \\
\text { Count-N, Cuprofix } \\
\text { Disperss, Kocide, } \\
\text { Nordox, Nu Cop, }\end{array}$ & M1 & & & & $\begin{array}{l}\text { Bacterial blight } \\
\text { Downy mildew } \\
\text { Purple blotch }\end{array}$ & See the individual labels \\
\hline $\begin{array}{l}\text { Iprodione 4L (ipro- } \\
\text { dione) }\end{array}$ & 2 & $1.5 \mathrm{pt}$ & $7.5 \mathrm{pt}$ & 7 & $\begin{array}{l}\text { Botrytis } \\
\text { Purple blotch }\end{array}$ & \\
\hline $\begin{array}{l}\text { Scala SC (pyrimeth- } \\
\text { anil) }\end{array}$ & 9 & $18 \mathrm{oz}$ & $54 \mathrm{oz}$ & 7 & $\begin{array}{l}\text { Botrytis } \\
\text { Purple blotch }\end{array}$ & \\
\hline $\begin{array}{l}\text { Switch 625WG } \\
\text { (cyprodinil/fludioxo- } \\
\text { nil) }\end{array}$ & $9+12$ & $14 \mathrm{oz}$ & $56 \mathrm{oz}$ & 7 & $\begin{array}{l}\text { Botrytis } \\
\text { Purple blotch }\end{array}$ & $\begin{array}{l}\text { After } 2 \text { appl. of Switch, a/w with } \\
\text { another fungicide with a different } \\
\text { mode of action for } 2 \text { applications }\end{array}$ \\
\hline $\begin{array}{l}\text { Serenade Max (bio- } \\
\text { fungicide) (QST } 713 \\
\text { strain of Bacillus } \\
\text { subtilis) }\end{array}$ & & $3 \mathrm{lb}$ & & 0 & $\begin{array}{l}\text { Botrytis } \\
\text { Downy mildew Purple } \\
\text { blotch } \\
\text { Rust }\end{array}$ & \\
\hline $\begin{array}{l}\text { Sonata (biofungi- } \\
\text { cide) (QST } 2808 \\
\text { strain of Bacillus } \\
\text { pumilis) }\end{array}$ & & $4 \mathrm{qt}$ & & 0 & $\begin{array}{l}\text { Downy mildew Powdery } \\
\text { mildew Rust }\end{array}$ & \\
\hline $\begin{array}{l}\text { Topsin M 70WDG } \\
\text { (other formulations } \\
\text { available) (thiophan- } \\
\text { ate-methyl) }\end{array}$ & 1 & $2 \mathrm{lb}$ & & & White rot & $\begin{array}{l}\text { Spray in open furrows at seed- } \\
\text { ing. Not to be used in any irriga- } \\
\text { tion system }\end{array}$ \\
\hline $\begin{array}{l}\text { Ultra Flourish 2EC } \\
\text { (mefenoxam) }\end{array}$ & 4 & $\begin{array}{l}2 \mathrm{pt} / \mathrm{trtd} \\
\text { acre }\end{array}$ & & & Pythium damping-off & Soil treatment at planting only \\
\hline \multicolumn{7}{|c|}{116.8 qts. for green onions } \\
\hline \multicolumn{7}{|c|}{$221.0 \mathrm{lbs}$. for green onions } \\
\hline \multicolumn{7}{|c|}{322.4 lbs. for green onions } \\
\hline \multicolumn{7}{|c|}{43 pts. for green bunching, leeks, shallots \& garlic } \\
\hline \multicolumn{7}{|c|}{514 days for green bunching, leeks, shallots \& garlic } \\
\hline $62.7 \mathrm{lbs}$. for garlic & & & & & & \\
\hline
\end{tabular}


Table 6. Selected insecticides approved for use on insects attaching onions and allies.

\begin{tabular}{|c|c|c|c|c|c|c|}
\hline $\begin{array}{l}\text { Trade Name } \\
\text { (Common Name) }\end{array}$ & $\begin{array}{l}\text { Rate } \\
\text { (product/acre) }\end{array}$ & $\begin{array}{l}\text { REl } \\
\text { (hours) }\end{array}$ & $\begin{array}{l}\text { Days to } \\
\text { Harvest }\end{array}$ & Insects & $\begin{array}{l}\text { MOA } \\
\text { Code }^{1}\end{array}$ & Notes \\
\hline $\begin{array}{l}\text { Agree WG } \\
\text { (Bacillus thuringiensis } \\
\text { subspecies aizawai) }\end{array}$ & $1.0-2.0 \mathrm{lb}$ & 4 & 0 & $\begin{array}{l}\text { lepidopteran larvae (cater- } \\
\text { pillar pests) }\end{array}$ & $11 \mathrm{~B} 1$ & $\begin{array}{l}\text { Apply when larvae are small for } \\
\text { best control. } \\
\text { OMRI-listed². }\end{array}$ \\
\hline $\begin{array}{l}{ }^{*} \text { Ambush } 25 \text { W } \\
\text { (permethrin) } \\
\text { (dry only) }\end{array}$ & $6.4-19.2 \mathrm{oz}$ & 12 & 1 & $\begin{array}{l}\text { armyworms, cutworms, } \\
\text { leafminers, onion mag- } \\
\text { got (adults), onion thrips, } \\
\text { stink bugs }\end{array}$ & 3 & $\begin{array}{l}\text { Dry bulb only and garlic. } \\
\text { Maximum of } 128 \text { oz/acre per } \\
\text { season. }\end{array}$ \\
\hline $\begin{array}{l}{ }^{*} \text { Ammo } 2.5 \mathrm{EC} \\
\text { (cypermethrin) }\end{array}$ & $2.0-5.0 \mathrm{fl} \mathrm{oz}$ & 12 & 7 & $\begin{array}{l}\text { aphids, armyworms, cut- } \\
\text { worms, leafminers, onion } \\
\text { maggot adults, stink bugs }\end{array}$ & 3 & $\begin{array}{l}\text { All Allium spp., green and dry. } \\
\text { Maximum of } 25 \text { oz product/acre } \\
\text { per season. }\end{array}$ \\
\hline $\begin{array}{l}\text { Aza-Direct } \\
\text { (azadirachtin) }\end{array}$ & $\begin{array}{l}1-2 \text { pts, up to } \\
3.5 \text {, if needed }\end{array}$ & 4 & 0 & $\begin{array}{l}\text { aphids, beetles, caterpil- } \\
\text { lars, leafhoppers, leafmin- } \\
\text { ers, mites, stink bugs, } \\
\text { thrips, weevils, whiteflies }\end{array}$ & 26 & $\begin{array}{l}\text { Antifeedant, repellant, insect } \\
\text { growth regulator. OMRI-listed². }\end{array}$ \\
\hline $\begin{array}{l}\text { Azatin XL } \\
\text { (azadirachtin) }\end{array}$ & $5-21 \mathrm{fl} \mathrm{oz}$ & 4 & 0 & $\begin{array}{l}\text { aphids, beetles, caterpil- } \\
\text { lars, leafhoppers, leafmin- } \\
\text { ers, thrips, weevils, } \\
\text { whiteflies }\end{array}$ & 26 & $\begin{array}{l}\text { Antifeedant, repellant, insect } \\
\text { growth regulator. }\end{array}$ \\
\hline $\begin{array}{l}\text { Biobit HP } \\
\text { (Bacillus thuringiensis } \\
\text { subspecies kurstaki) }\end{array}$ & $0.5-2.0 \mathrm{lb}$ & 4 & 0 & $\begin{array}{l}\text { caterpillars (will not con- } \\
\text { trol large armyworms) }\end{array}$ & $11 \mathrm{~B} 2$ & $\begin{array}{l}\text { Treat when larvae are young. } \\
\text { Good coverage is essential. Can } \\
\text { be used in the greenhouse. } \\
\text { OMRI-listed². }\end{array}$ \\
\hline $\begin{array}{l}\text { BotaniGard } 22 \text { WP, ES } \\
\text { (Beauveria bassiana) }\end{array}$ & $\begin{array}{l}\text { WP: } \\
0.5-2 \mathrm{lb} / 100 \mathrm{gal} \\
\text { ES: } \\
0.5-2 \text { qts/100 } \\
\text { gal }\end{array}$ & 4 & 0 & aphids, thrips, whiteflies & -- & $\begin{array}{l}\text { May be used in greenhouses. } \\
\text { Contact dealer for recommen- } \\
\text { dations if an adjuvant must be } \\
\text { used. Not compatible in tank mix } \\
\text { with fungicides. }\end{array}$ \\
\hline $\begin{array}{l}\text { Crymax WDG } \\
\text { (Bacillus thuringiensis } \\
\text { subspecies kurstaki) }\end{array}$ & $0.5-2.0 \mathrm{lb}$ & 4 & 0 & caterpillars & $11 \mathrm{~B} 2$ & $\begin{array}{l}\text { Use high rate for armyworms. } \\
\text { Treat when larvae are young. }\end{array}$ \\
\hline $\begin{array}{l}\text { Deliver } \\
\text { (Bacillus thuringiensis } \\
\text { subspecies kurstaki) }\end{array}$ & $0.25-1.5 \mathrm{lb}$ & 4 & 0 & caterpillars & $11 \mathrm{~B} 2$ & $\begin{array}{l}\text { Use higher rates for armyworms. } \\
\text { OMRI-listed?2. }\end{array}$ \\
\hline \multirow[t]{2}{*}{$\begin{array}{l}\text { *Diazinon AG500, } \\
\text { 4EC, *50W } \\
\text { (diazinon) }\end{array}$} & $\begin{array}{l}\text { foliar - } \\
\text { AG500, 4EC: } \\
1 \mathrm{pt} \\
\text { 50W: } 1 \mathrm{lb}\end{array}$ & 24 & 14 & onion thrips & $1 \mathrm{~B}$ & Bulb and green \\
\hline & $\begin{array}{l}\text { preplant - } \\
\text { AG500, 4EC: 3- } \\
4 \text { qts } \\
50 W: 6-8 \mathrm{lb}\end{array}$ & 24 & preplant & wireworms & 1B & See label. \\
\hline $\begin{array}{l}\text { DiPel DF } \\
\text { (Bacillus thuringiensis } \\
\text { subspecies kurstaki) }\end{array}$ & $0.5-2.0 \mathrm{lb}$ & 4 & 0 & caterpillars & $11 \mathrm{~B} 2$ & $\begin{array}{l}\text { Treat when larvae are young. } \\
\text { Good coverage is essential. } \\
\text { OMRI-listed?2. }\end{array}$ \\
\hline $\begin{array}{l}\text { Entrust } \\
\text { (spinosad) }\end{array}$ & $1-2.50 z$ & 4 & 1 & $\begin{array}{l}\text { armyworms, dipteran } \\
\text { leafminers, flea beetle, } \\
\text { loopers, suppression of } \\
\text { thrips }\end{array}$ & 5 & $\begin{array}{l}\text { No more than } 5 \text { applications per } \\
\text { year ( } 9 \text { oz product). }\end{array}$ \\
\hline $\begin{array}{l}\text { Extinguish } \\
\text { ((S)-methoprene) }\end{array}$ & $1-1.5 \mathrm{lb}$ & 4 & 0 & fire ants & $7 \mathrm{~A}$ & $\begin{array}{l}\text { Slow-acting IGR (insect growth } \\
\text { regulator). Best applied early } \\
\text { spring and fall where crop will be } \\
\text { grown. Colonies will be reduced } \\
\text { after three weeks and eliminated } \\
\text { after } 8 \text { to } 10 \text { weeks. May be } \\
\text { applied by ground equipment or } \\
\text { aerially. }\end{array}$ \\
\hline
\end{tabular}


Table 6. Continued.

\begin{tabular}{|c|c|c|c|c|c|c|}
\hline $\begin{array}{l}\text { Trade Name } \\
\text { (Common Name) }\end{array}$ & $\begin{array}{l}\text { Rate } \\
\text { (product/acre) }\end{array}$ & $\begin{array}{l}\text { REI } \\
\text { (hours) }\end{array}$ & $\begin{array}{l}\text { Days to } \\
\text { Harvest }\end{array}$ & Insects & $\begin{array}{l}\text { MOA } \\
\text { Code }^{1}\end{array}$ & Notes \\
\hline $\begin{array}{l}\text { Javelin WG } \\
\text { (Bacillus thuringiensis } \\
\text { subspecies kurstaki) }\end{array}$ & $0.12-1.5 \mathrm{lb}$ & 4 & 0 & $\begin{array}{l}\text { most caterpillars, but } \\
\text { not Spodoptera species } \\
\text { (armyworms) }\end{array}$ & $11 \mathrm{~B} 2$ & $\begin{array}{l}\text { Treat when larvae are young. } \\
\text { Thorough coverage is essential. } \\
\text { OMRI-listed }{ }^{2} \text {. }\end{array}$ \\
\hline $\begin{array}{l}\text { Knack } \\
\text { (pyriproxyfen) }\end{array}$ & $8 \mathrm{fl} \mathrm{oz}$ & 12 & 3 & $\begin{array}{l}\text { onion thrips, western } \\
\text { flower thrips }\end{array}$ & $7 \mathrm{D}$ & $\begin{array}{l}\text { Maximum of } 2 \text { applications, at } \\
\text { least } 14 \text { days apart. }\end{array}$ \\
\hline $\begin{array}{l}{ }^{*} \text { Lannate LV; *SP } \\
\text { (methomyl) }\end{array}$ & $\begin{array}{l}\text { LV: } 1.5-3.0 \mathrm{pt} \\
\text { SP: } 0.5-1.0 \mathrm{lb}\end{array}$ & 48 & $\begin{array}{l}7=(\text { dry } \\
\text { and } \\
\text { green) }\end{array}$ & $\begin{array}{l}\text { beet armyworm, black cut- } \\
\text { worm, thrips, variegated } \\
\text { cutworm }\end{array}$ & $1 \mathrm{~A}$ & $\begin{array}{l}\text { Add a wetting agent to improve } \\
\text { coverage. }\end{array}$ \\
\hline $\begin{array}{l}\text { Lepinox WDG } \\
\text { (Bacillus thuringiensis } \\
\text { subspecies kurstaki) }\end{array}$ & $1.0-2.0 \mathrm{lb}$ & 12 & 0 & $\begin{array}{l}\text { for most caterpillars, } \\
\text { including beet armyworm } \\
\text { (see label) }\end{array}$ & 11B2 & $\begin{array}{l}\text { Treat when larvae are small. } \\
\text { Thorough coverage is essential. }\end{array}$ \\
\hline $\begin{array}{l}\text { Lorsban } 15 \text { G; } 75 W G \\
\text { (chlorpyrifos) }\end{array}$ & $\begin{array}{l}\text { See labels for } \\
\text { rates }\end{array}$ & 24 & $\begin{array}{l}\text { at plant- } \\
\text { ing }\end{array}$ & onion maggot & $1 \mathrm{~B}$ & Dry bulb only. \\
\hline $\begin{array}{l}\text { Malathion 8F } \\
\text { (malathion) }\end{array}$ & $1-2 \mathrm{pt}$ & 12 & 3 & onion maggot, thrips & $1 \mathrm{~B}$ & \\
\hline $\begin{array}{l}\text { M-Pede } \mathbf{4 9 \%} \text { EC } \\
\text { Soap, insecticidal }\end{array}$ & $1-2 \%$ V/V & 12 & 0 & $\begin{array}{l}\text { aphids, leafhoppers, } \\
\text { mites, plant bugs, thrips, } \\
\text { whiteflies }\end{array}$ & -- & OMRI-listed². \\
\hline $\begin{array}{l}\text { *Mustang Max } \\
\text { (zeta-cypermethrin) }\end{array}$ & $2.24-4.0 \mathrm{oz}$ & 12 & 7 & $\begin{array}{l}\text { aphids, armyworms, cut- } \\
\text { worms, leafminers, onion } \\
\text { maggot adults, onion } \\
\text { thrip, stink bugs }\end{array}$ & 3 & \\
\hline $\begin{array}{l}\text { Neemix } 4.5 \text { EC } \\
\text { (azadirachtin) }\end{array}$ & $4-16 \mathrm{fl} \mathrm{oz}$ & 12 & 0 & $\begin{array}{l}\text { aphids, armyworms, cab- } \\
\text { bage looper, cutworms, } \\
\text { leafminers, onion maggot, } \\
\text { thrips, whiteflies }\end{array}$ & 26 & OMRI-listed ${ }^{2}$ \\
\hline $\begin{array}{l}\text { *Penncap-M } \\
\text { (methyl parathion) }\end{array}$ & $2 \mathrm{pt}$ & $\begin{array}{l}4 \text { days - } \\
\text { See } \\
\text { label }\end{array}$ & 15 & thrips & $1 \mathrm{~B}$ & $\begin{array}{l}\text { Do not apply when onions are } \\
\text { blooming and bees are foraging. }\end{array}$ \\
\hline $\begin{array}{l}\text { *Pounce } 3.2 \text { EC } \\
\text { (permethrin) }\end{array}$ & $4-12$ oz & 12 & 1 & $\begin{array}{l}\text { armyworms, cutworms, } \\
\text { leafminers, onion maggot, } \\
\text { stink bugs, thrips }\end{array}$ & 3 & \\
\hline $\begin{array}{l}\text { Pyrellin EC } \\
\text { (pyrethrin + rotenone) }\end{array}$ & $1-2 \mathrm{pt}$ & 12 & $\begin{array}{l}12 \\
\text { hours }\end{array}$ & $\begin{array}{l}\text { aphids, leafhoppers, loop- } \\
\text { ers, mites, plant bugs, } \\
\text { stink bugs, thrips, white- } \\
\text { flies }\end{array}$ & 3,21 & \\
\hline $\begin{array}{l}\text { *Telone C-35 (dichlo- } \\
\text { ropropene + chloro- } \\
\text { picrin) } \\
{ }^{*} \text { Telone II } \\
\text { (dichloropropene) }\end{array}$ & See label & $\begin{array}{l}5 \text { days - } \\
\text { See } \\
\text { label }\end{array}$ & preplant & symphylans, wireworms & -- & $\begin{array}{l}\text { See supplemental label for use } \\
\text { restrictions in south and central } \\
\text { Florida. }\end{array}$ \\
\hline $\begin{array}{l}\text { Trigard } \\
\text { (cyromazine) }\end{array}$ & $2.66 \mathrm{oz}$ & 12 & 7 & leafminers & 17 & $\begin{array}{l}\text { Do not make more than } 6 \text { appli- } \\
\text { cations. }\end{array}$ \\
\hline $\begin{array}{l}\text { Trilogy } \\
\text { (extract of neem oil) }\end{array}$ & $0.5-2.0 \% \mathrm{~V} / \mathrm{V}$ & 4 & 0 & $\begin{array}{l}\text { aphids, mites, suppression } \\
\text { of thrips and whiteflies }\end{array}$ & 26 & $\begin{array}{l}\text { Apply morning or evening to } \\
\text { reduce potential for leaf burn. } \\
\text { Toxic to bees exposed to direct } \\
\text { treatment. } \\
\text { OMRI-listed } 2 \text {. }\end{array}$ \\
\hline
\end{tabular}


Table 6. Continued.

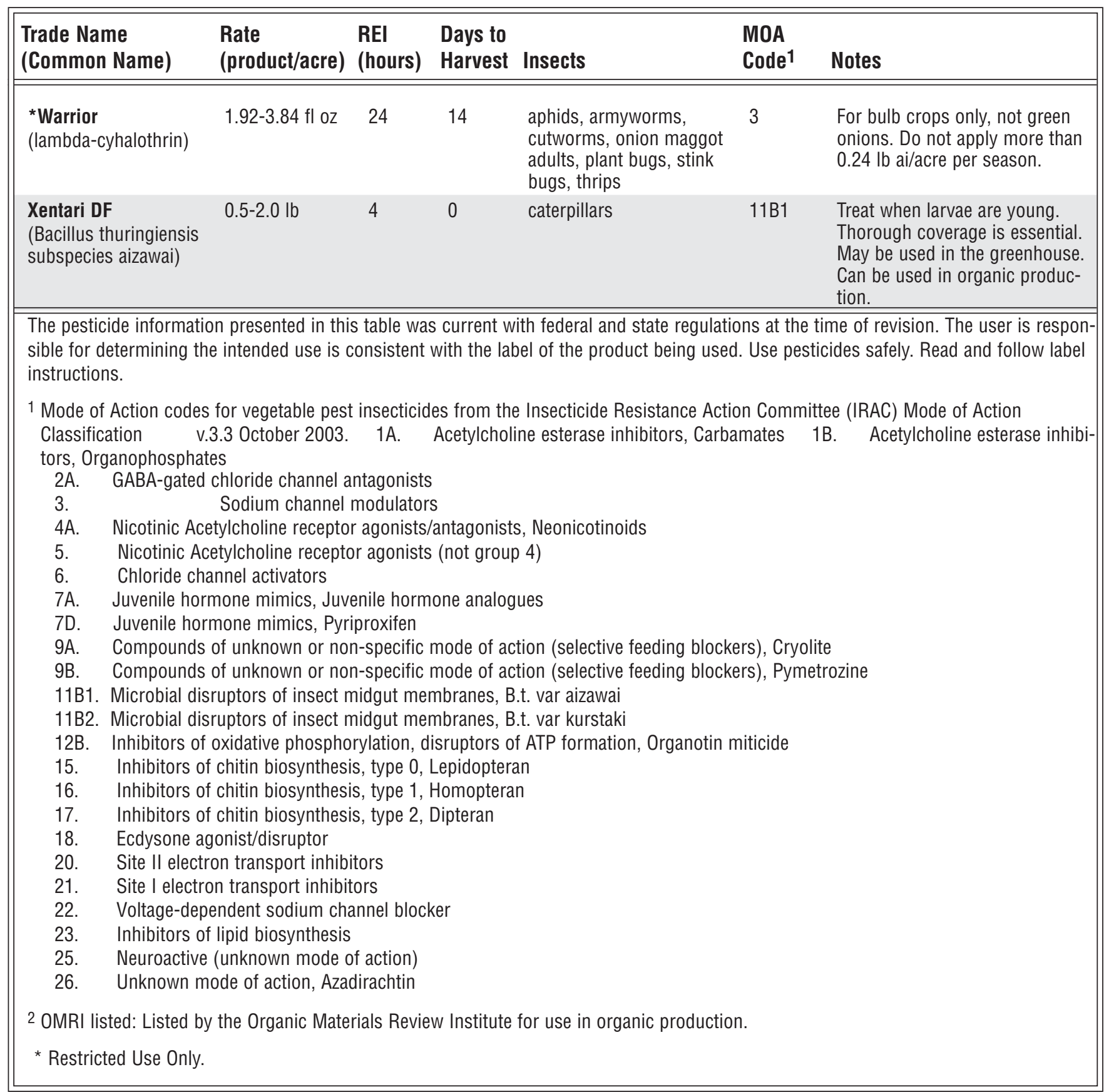

